\title{
Eggshell Calcification of Kidney in Ureteropelvic Junction Obstruction
}

\author{
Narmada P. Gupta, Rajiv Yadav \\ Department of Urology, All India Institute of Medical Sciences, New Delhi, India
}

\begin{abstract}
Eggshell calcification of kidney in case of ureteropelvic junction obstruction (UPJO) is an uncommon finding with only a few cases reported in literature. We report a thirty-year-old symptomatic man with curvilinear calcification in hydronephrotic right kidney. Thorough investigations to rule out genitourinary tuberculosis and hydatid disease of kidney were performed prior to the definitive management by laparoscopic approach.
\end{abstract}

Key words: ureter; hydronephrosis; kidney pelvis; calcinosis

Int Braz J Urol. 2006; 32: 557-9

\section{INTRODUCTION}

Eggshell calcification of kidney in case of ureteropelvic junction obstruction (UPJO) is an uncommon finding. Such kind of curvilinear calcification is more commonly found in benign cysts, renal cell carcinoma and hydatid cyst. We report a thirty-year-old symptomatic man with eggshell calcification in hydronephrotic right kidney. Progressive deposition of calcium salts in a chronically dilated renal parenchyma may have caused calcification in the hyalinized fibrous renal tissue.

\section{CASE REPORT}

A thirty-year-old man was investigated for abdominal pain and a palpable mass in the right lumbar region. He had no associated urinary symptoms or bowel dysfunction. The only significant history was a pulmonary tuberculosis ten years before for which he was adequately treated. He had no history of any operative intervention or trauma in the past. On physical examination, he was normotensive and had a $7 \mathrm{~cm}$ hard, nontender bimanually palpable lump with restricted mobility in the right lumbar and umbilical region. No bruit was present over the lump. An investigation revealed a normal urine routine and microscopic examination, sterile urine culture and a normal hemogram. Serum creatinine, blood urea nitrogen and serum calcium were unremarkable. Urine for acid-fast bacillus microscopy was negative thrice and urine polymerase chain reaction for Mycobacterium tuberculosis was also negative. Indirect hemagglutination test for hydatid was negative. KUB film revealed an eggshell like calcification in the right kidney region (Figure-1). Subsequent ultrasound and computerized tomography showed a well defined, cystic right kidney (Figure$2)$. Renal scan confirmed the absence of any functional renal parenchyma on the right side. On 
cystoscopy, the right orifice was normal and no urine flow was detected. Right retrograde pyelogram revealed a thin ureter, which was obstructed at the ureteropelvic junction. Laparoscopic nephrectomy was done. Multiple sections examined showed a fibrocollagenous cystic wall with hyalinization, calcification and ossification. Certain areas with intact epithelial lining and thyroidization of renal tubules were also observed. No granulomatous inflammation or parasite was present.

\section{COMMENTS}

Calcification of kidney associated to ureteropelvic junction obstruction (UPJO) is rare and only a few cases have been reported in literature (1). Although such eggshell calcification is usually observed in benign condition, surgical intervention may still be indicated as the risk of malignancy in such lesion is $20 \%$ (2). Interestingly, Gold et al have

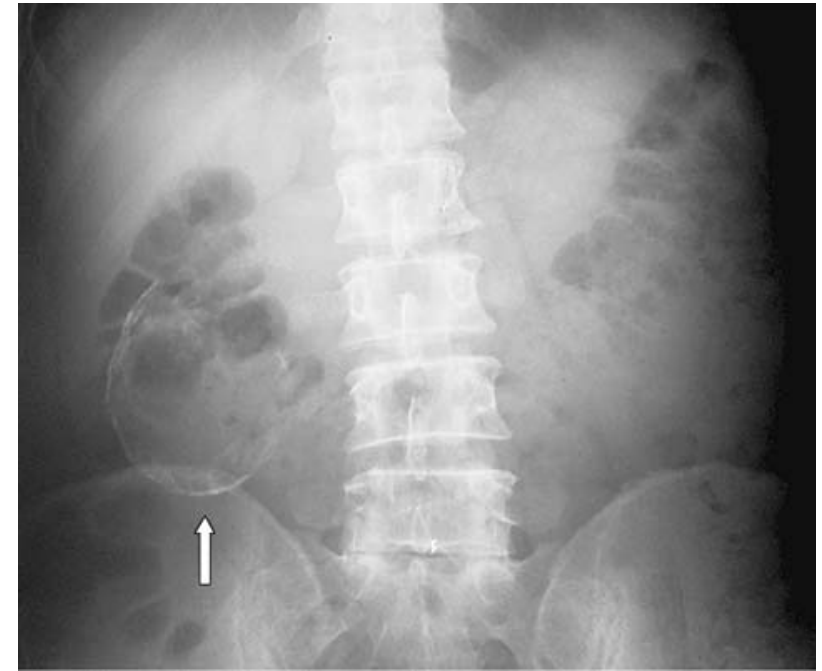

Figure 1 - Kidneys, ureters and bladder X-ray showing eggshell calcification in the region of right kidney.

described a case of UPJO with a calcified renal pelvis and superimposed spindle urothelial carcinoma (3).
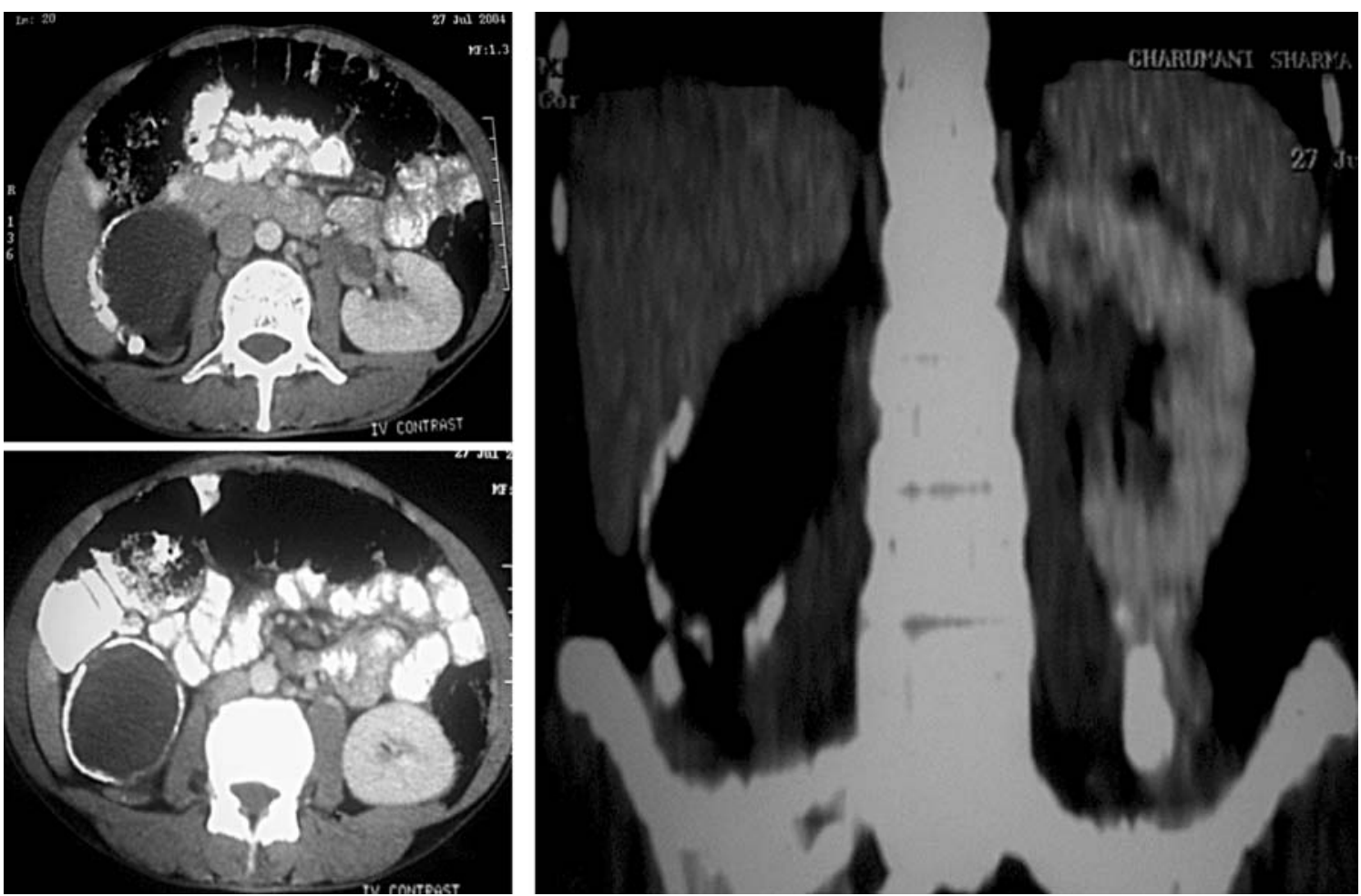

Figure 2 - Computerized tomography showing calcified right hydronephrotic kidney. 
Calcification in this situation occurred despite normal serum levels of calcium probably due to progressive deposition of calcium salts in damaged and hyalinized tissue in the wall of chronic hydronephrotic kidney.

\section{CONFLICT OF INTEREST}

None declared.

\section{REFERENCES}

1. Tsujimura A, Imazu T, Nishimura K, Sugao H, Oka T, Takaha M, et al.: Ureteropelvic junction obstruction with renal pelvic calcification: a case report. J Urol. 1993; 150: 1889-90.

2. Daniel WW Jr, Hartman GW, Witten DM, Farrow GM, Kelalis PP: Calcified renal masses. A review of ten years experience at the Mayo Clinic. Radiology. 1972; 103: 503-8.

3. Gold RP, Saitas V, Pellman C: Congenital ureteropelvic junction obstruction with calcified renal pelvis and superimposed spindle cell urothelial carcinoma. Urol Radiol. 1990; 12: 15-7.

\section{Correspondence address:}

Dr. Narmada P Gupta

Head, Department of Urology

All India Institute of Medical Sciences

Ansari Nagar, New Delhi 110029, INDIA

Fax: + 9111 686-2663

E-mail: narmadagupta@hotmail.com 